\title{
Colecistectomía subtotal: una alternativa en el manejo de la colecistectomía difícil
}

\section{Subtotal cholecystectomy: an alternative in the management of difficult cholecystectomy}

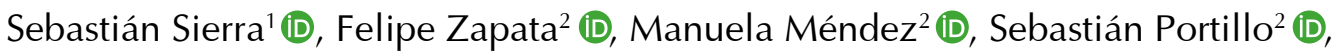 \\ Camilo Restrepo ${ }^{3}$, \\ Médico, especialista en Cirugía General, Clínica CES; profesor Departamento de Cirugía, Universidad de Antioquia, Medellín, \\ Colombia. \\ Médico, especialista en Cirugía General, Medellín, Colombia. \\ 3 Médico, residente Cirugía General, Universidad CES, Medellín, Colombia.
}

\section{Resumen}

Introducción. Alrededor de un Io \% de las laparoscopias se convierten a cirugía abierta por dificultades en obtener una visión crítica durante la colecistectomía en colecistitis severas. La colecistectomía subtotal es una posibilidad terapéutica disponible, que disminuye la tasa de conversión en cirugía laparoscópica y mantiene bajas tasas de morbilidad y mortalidad.

Métodos. Estudio descriptivo, retrospectivo, de pacientes sometidos a colecistectomía subtotal en la Clínica CES (Medellín, Colombia) entre enero y diciembre de 20I5. Se identificaron variables demográficas, detalles de la cirugía, morbilidad y mortalidad.

Resultados. De un total de 7 IO colecistectomías en dicho periodo, a I7 (2,4 \%) se les realizó colecistectomía subtotal. Quince $(88 \%)$ de ellas fueron por laparoscopia y dos requirieron conversión. La distribución en cuanto a sexo fue similar (Io mujeres / 7 hombres) y la edad promedio fue de 5I años. El tiempo quirúrgico promedio fue de II9 minutos. En I4 (82 \%) pacientes se dejó drenaje subhepático. Dos pacientes presentaron fístula biliar y un paciente reingresó por un hematoma; no se presentaron otras complicaciones. La estancia hospitalaria promedio fue de 5,2 días.

Discusión. La colecistectomía subtotal es una alternativa en pacientes con colecistectomía difícil y en nuestra experiencia presenta una alta tasa de éxito.

Palabras clave: colecistitis aguda; colecistectomía laparoscópica; complicaciones; conversión a cirugía abierta.

Fecha de recibido: 13/02/2020 - Fecha de aceptación: 22/02/2020

Correspondencia: Sebastián Sierra, Calle 58 \# 50C - 2, Prado, Clínica CES, Medellín, Colombia. Teléfono: 034-5767273.

sebastiancirugia@gmail.com

Citar como: Sierra S, Zapata F, Méndez M, Portillo S, Restrepo C. Colecistectomía subtotal: una alternativa en el manejo de la colecistectomía difícil. Rev Colomb Cir. 2020;35:593-600. https://doi.org/10.30944/20117582.565

Este es un artículo de acceso abierto bajo una Licencia Creative Commons - BY-NC-ND https://creativecommons.org/licenses/by-nc$\mathrm{nd} / 4.0 /$ deed.es 


\begin{abstract}
Introduction. About $10 \%$ of laparoscopies are converted to open surgery due to difficulties in obtaining critical vision during cholecystectomy in severe cholecystitis. Subtotal cholecystectomy is an available therapeutic possibility, which decreases the conversion rate in laparoscopic surgery and maintains low morbidity and mortality rates.

Methods. Descriptive, retrospective study of patients who underwent subtotal cholecystectomy between January and December 2015. Demographic variables, details of surgery, morbidity and mortality were identified.
\end{abstract}

Results. Of a total of 7 IO cholecystectomies in that period, $\mathrm{I} 7$ (2.4\%) underwent subtotal cholecystectomy. Fifteen (88\%) of them were by laparoscopy and two required conversion. The gender distribution was similar (IO women/7 men) and the average age was 5 I years. The average surgical time was II9 minutes. Subhepatic drainage was left in I4 $(\mathbf{8 2} \%)$ patients. Two patients had a biliary fistula and one patient was readmitted for a hematoma; there were no other complications. The average hospital stay was 5.2 days.

Discussion. Subtotal cholecystectomy is an alternative in patients with difficult cholecystectomy and in our experience, it has a high success rate.

Keywords: acute cholecystitis; cholecystectomy, laparoscopic; complications; conversion to open surgery.

\section{Introducción}

La colelitiasis y su principal complicación, la colecistitis, son enfermedades con las cuales el cirujano se enfrenta en su día a día. Se conoce que aproximadamente el IO a I $5 \%$ de la población en los Estados Unidos de América tiene cálculos en la vesícula. En Colombia, pese a no contar con estadísticas específicas, pareciera que esta cifra es similar ${ }^{1,2}$.

Entre las diferentes manifestaciones clínicas, se encuentran el cólico biliar, la colecistitis aguda, subaguda o crónica, la coledocolitiasis, la colangitis y la pancreatitis, entre otros ${ }^{3,4}$. El estándar de oro en el manejo continúa siendo la colecistectomía laparoscópica, porque tiene una menor tasa de complicaciones, recuperación más pronta de los pacientes y menor estancia hospitalaria ${ }^{5,6}$.

En aproximadamente un $90 \%$ de los pacientes que son llevados a colecistectomía laparoscópica, se logra concluir la cirugía por dicha vía. El ıo \% restante presentarán algún tipo de complicación de la enfermedad que no permite continuar por mínima invasión y se requiere convertir a cirugía por vía abierta. Entre las causas más frecuentes se encuentran la alteración de la anatomía por el proceso inflamatorio, presencia de adherencias, sangrado incontrolable y la co- ledocolitiasis concomitante. La principal causa de conversión continúa siendo la imposibilidad de obtener una adecuada visión crítica de seguridad durante el procedimiento quirúrgico, por la severa inflamación sobre el triángulo de Calot (figura I), lo que lleva a un aumento significativo en la incidencia de lesión de la vía biliar cuando se procede a realizar la colecistectomía, bien sea laparoscópica o abierta ${ }^{\text {7-10 }}$.

Son situaciones como esta, en la que se ha acuñado el término "colecistectomía difícil" ". En dichos casos, será decisión del cirujano determinar qué paso tomar, según las circunstancias en las que se encuentre, por estado del paciente y su anatomía, capacidad del ayudante, habili-

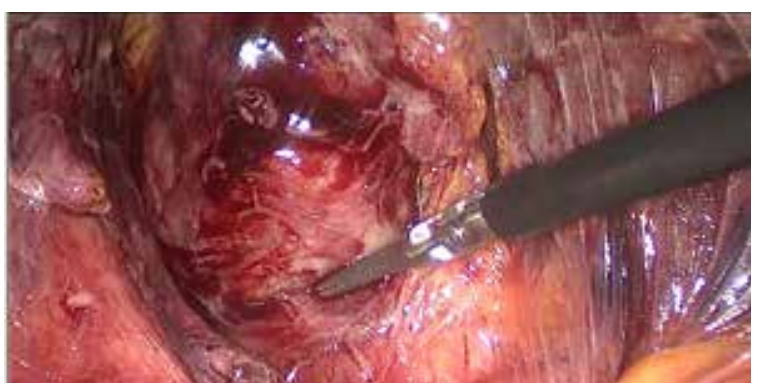

Figura 1. Colecistectomía difícil. Las estructuras del triángulo de Calot, no son identificables. La pinza señala la bolsa de Hartman. 
dades propias, o equipo disponible, entre otras. Así como la conversión a cirugía abierta es una alternativa válida, la posibilidad de continuar por vía mínimamente invasiva para conservar sus beneficios, es también posible, por lo que la colecistectomía subtotal laparoscópica es una opción adecuada.

La primera descripción de una colecistectomía subtotal abierta se realizó en I898 por Hans $\mathrm{Kehr}{ }^{9}$. Desde entonces ha habido múltiples modificaciones de la técnica quirúrgica, más aún con la aparición de la cirugía laparoscópica. Recientemente, Steven Strasberg, reconocido cirujano en el área de la cirugía hepatobiliar, propuso una puesta en común de la nomenclatura del procedimiento ${ }^{9}$. Viendo la dificultad que presentaban los términos utilizados hasta el momento y la confusión que pueden generar, divide aquellas cirugías donde se extrae más de la mitad de la vesícula, pero no el órgano en su totalidad, en dos: colecistectomía subtotal reconstructiva y colecistectomía subtotal fenestrada, diferenciadas por el hecho de crear o no un remanente de vesícula, respectivamente ${ }^{9, \mathrm{IO}}$.

\section{Métodos}

Se realizó un estudio descriptivo, retrospectivo, de corte transversal, cuyo objetivo principal fue describir las indicaciones y complicaciones de la colecistectomía subtotal en la clínica CES, centro de referencia de la patología biliar en Medellín, Colombia. Los objetivos secundarios fueron describir la técnica quirúrgica, conocer los motivos que llevaron a tomar esta decisión e identificar la necesidad de procedimientos adicionales durante su seguimiento.

Se revisaron las historias clínicas de los pacientes mayores de 18 años que ingresaron a la clínica CES entre enero y diciembre de 2015 con patología biliar y que hubieran sido sometidos a colecistectomía abierta o laparoscópica, con un seguimiento a los 30 días y 2 años. De estos se filtraron aquellos a quienes se les realizó una colecistectomía subtotal por cualquier abordaje, teniendo como referencia el libro de estadística del servicio de cirugía. La recolección de datos inició previa aprobación del proyecto de investigación por el comité de ética de la Clínica CES.

Se excluyeron los pacientes con patología maligna de vesícula biliar conocida antes de la cirugía, o con datos insuficientes en la historia clínica.

\section{Resultados}

De los 7io pacientes que fueron llevados a colecistectomía en la Clínica CES (Medellín, Colombia) entre enero y diciembre de 20I5, a I7 (2,39\%) se les practicó una colecistectomía subtotal. Ninguno de ellos cumplió criterios de exclusión, por lo que todos fueron incluidos en el análisis.

La distribución por género fue similar, con Io (58,8 \%) mujeres y 7 (4I,I \%) hombres, y la edad promedio fue de 5I años (rango: 25-8I años). Quince pacientes $(88,2 \%)$ se operaron en un contexto urgente y dos (II,7 \%) fueron cirugías electivas. Los pacientes urgentes, tenían una evolución de los síntomas en promedio de 6,9 días y los electivos de 4 meses. Cinco pacientes $(29,4 \%)$ eran ASA I, nueve (52,9\%) ASA II, tres (I7,6\%) ASA III y ninguno ASA IV o V.

El principal hallazgo quirúrgico que motivó la realización de colecistectomía subtotal fue la inflamación alrededor del triángulo de Calot. Los detalles de la cirugía se resumen en la tabla I. Se

Tabla 1. Características de la cirugía en los pacientes sometidos a colecistectomía subtotal.

\begin{tabular}{lc}
\hline \multicolumn{1}{c}{ Características } & N (\%) \\
\hline Tiempo quirúrgico (minutos) & 119,5 \\
$\quad$ Promedio & $80-168$ \\
$\quad$ Rango & \\
Tipo de herida & $11(64,7 \%)$ \\
$\quad$ Contaminada & $6(35,2 \%)$ \\
$\quad$ Sucia & \\
Motivo de colecistectomía subtotal & $11(64,7 \%)$ \\
$\quad$ Inflamación del triángulo de Calot & $5(29,4 \%)$ \\
$\quad$ Síndrome de Mirizzi & $1(5,8 \%)$ \\
$\quad$ Vesícula esclero retráctil & \\
Uso de dren & $14(82,3 \%)$ \\
$\quad$ Si & $3(17,6 \%)$ \\
$\quad$ No & 11,6 \\
Tiempo drenaje (días) &
\end{tabular}


presentaron dos casos de conversión a cirugía abierta entre los I7 pacientes incluidos en el estudio (II,7 $\%$ ), ambos casos en pacientes obesos mórbidos, con inflamación severa que no permitía lograr la visión crítica por vía laparoscópica y llevaron al cirujano a tomar la decisión de convertir, y una vez en cirugía por vía abierta, se llevó a cabo una colecistectomía subtotal.

En las colecistectomías subtotales por laparoscopia ( $\mathrm{n}=\mathrm{I5}$ ) se encontraron descritas dos técnicas, una en donde se inicia la disección desde el fondo de la vesícula hasta llegar a la bolsa de Hartmann (fundocística), se hace apertura de la vesícula y sección distal a este nivel, con extracción completa de los cálculos (figura 2), lavado con solución salina hasta determinar que no hay más cálculos en su interior y cierre del muñón con sutura intracorpórea (figuras 3 y 4), y otra técnica, que realiza la disección de la vesícula de manera similar a la anterior, pero deja parte de la pared posterior de la vesícula en su lecho y fulgura la mucosa residual, buscando con esto reducir el sangrado profuso del lecho vesicular. En ningún caso se encontró una descripción de la técnica fenestrada.

En I4 de los I7 pacientes (82,3\%), I2 de cirugía laparoscópica y 2 de cirugía abierta, se recurrió al uso de drenaje subhepático, utilizando un dren cerrado con presión negativa. En ningún paciente se realizó colangiografía intraoperatoria. A ocho pacientes se les dio manejo antibiótico intrahospitalario según los hallazgos clínicos al ingreso y de la cirugía. El promedio de estancia hospitalaria fue de 5,2 días, siendo el promedio de la estancia preoperatoria de I,9 días y de la postoperatoria de 3,2 días.

Se envió el espécimen quirúrgico para estudio histopatológico en todos los casos, y demostró enfermedad inflamatoria aguda en el Ioo $\%$ y gangrena en el I $7 \%$; en ningún paciente se encontró malignidad.

Se hizo seguimiento en la historia clínica durante la hospitalización, reingresos y revisión ambulatoria por consulta externa (30 días postoperatorios). Se realizó adicionalmente un seguimiento a 2 años en la historia clínica para evaluar reingresos a la Clínica CES por motivos relacionados con su enfermedad biliar o complicaciones asociadas. Se presentó fístula biliar en dos pacientes, que requirieron algún tipo de intervención.

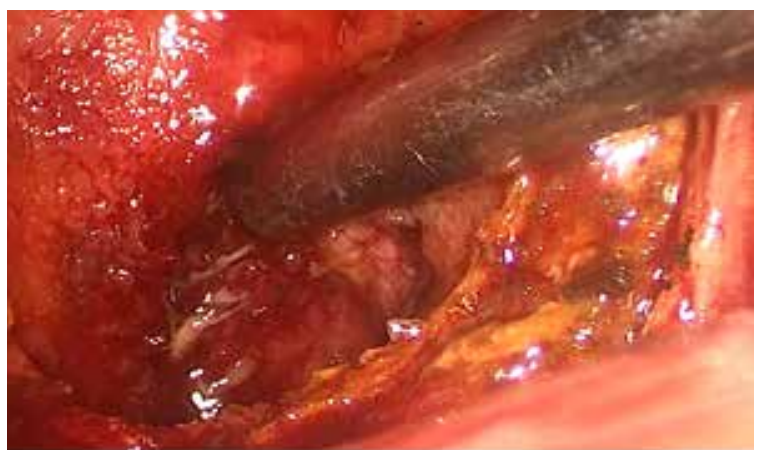

Figura 2. Momento de la cirugía en donde se muestra sección de la vesícula, inspección de su luz, extracción de cálculos e identificación del orificio del conducto cístico.

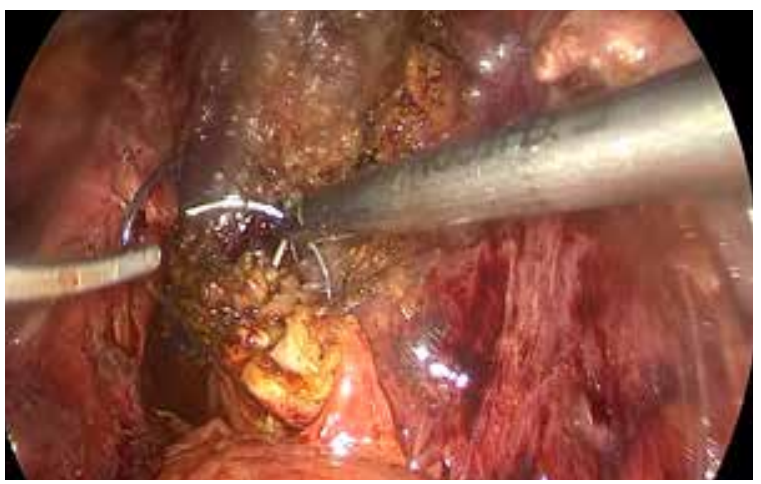

Figura 3. Sutura de las paredes vesiculares creando una "nueva vesícula" (técnica reconstructiva).

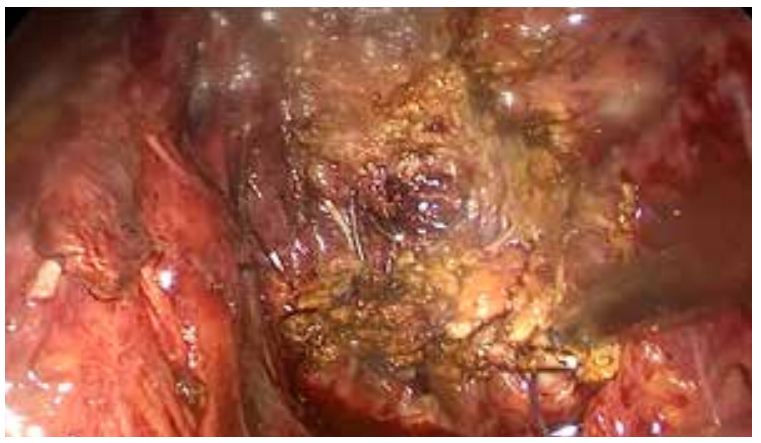

Figura 4. Aspecto final del nuevo muñón vesicular. Clip en lecho vesicular, con ligadura alta de la arteria cística. 
El primer paciente había sido llevado a cirugía laparoscópica urgente y convertido a cirugía abierta por el proceso inflamatorio local y gangrena asociada; en el postoperatorio presentó una fístula biliar de alto gasto, por lo que se ordenó una colangiopancreatografía retrógrada endoscópica (CPRE), la cual mostró fuga biliar por el muñón de la vesícula. Fue tratado de manera exitosa mediante esfinterotomía por esta vía y fue dado de alta dos días posterior al procedimiento. Finalmente, la fístula cerró y el dren se retiró 25 días después de la CPRE, durante la consulta médica ambulatoria de revisión.

El segundo paciente fue un caso programado para cirugía electiva, con colelitiasis sintomática, en quien se encontró un síndrome de Mirizzi por inflamación crónica y se le realizó una colecistectomía subtotal por laparoscopia. Su evolución postoperatoria fue tórpida, con fuga biliar de alto gasto, por lo que se llevó a una CPRE, sin hallazgos anormales. Entonces fue sometido a una reintervención por vía laparoscópica donde se encontró un bilioperitoneo generalizado, por filtración de bilis a nivel del muñón vesicular. El líquido fue aspirado y el muñón reforzado nuevamente con sutura. Posteriormente tuvo una adecuada evolución clínica, sin presencia de fugas biliares, y fue dado de alta dos días después de la reintervención.

Adicionalmente, un paciente presentó una colección subhepática, manejada como un hematoma postoperatorio, de forma expectante, sin intervenciones específicas. No hubo complicaciones extraabdominales ni muertes en el seguimiento descrito.

\section{Discusión}

Es bien sabido que la colecistectomía laparoscópica ofrece múltiples beneficios comparados con el procedimiento por vía abierta, tanto para el paciente como para los sistemas de salud. Sin embargo, en los pacientes con patología aguda, es frecuente encontrar inflamación severa, que hace el procedimiento laparoscópico más complejo ${ }^{12}$. La falta de visión crítica conlleva mayores tasas de conversión, bien sea para prevenir la lesión de vía biliar o para continuar la cirugía de reparo en el momento que esta se produce. Por eso, algunos autores han favorecido el uso de la colangiografía intraoperatoria de rutina, sin embargo, esto no ha demostrado reducir la tasa de lesión de vía biliar ya que hay aspectos técnicos a tener en cuenta, como es la oclusión del conducto cístico, que disminuye la eficacia de la misma ${ }^{13}$.

La colecistectomía subtotal laparoscópica es una opción a la hora de decidir si convertir o no la cirugía, y mantiene las ventajas de la cirugía mínimamente invasiva, como son la menor estancia hospitalaria y el tiempo de retorno a la actividad normal, y menos complicaciones infecciosas y no infecciosas comparadas con la técnica abierta. La lesión de vía biliar, descrita en la literatura con una incidencia del o,I al I,4 \%, puede ser mayor en casos urgentes y complicados (por ejemplo, gangrena, plastrón, colecistitis subaguda, etc.), al compararlo con la cirugía electiva, generalmente ambulatoria ${ }^{10,14,15}$. En nuestra serie de casos, ninguno de los pacientes presentó esa complicación durante el seguimiento realizado.

A pesar de que no hay una directriz dada por expertos que determine cuál es el mejor manejo para la colecistectomía difícil, la colecistectomía subtotal, por vía laparoscópica o abierta, continúa siendo una buena opción para el manejo. Dependerá de la experiencia del cirujano, en qué casos elegir cada una de las opciones disponibles, aunque continúa vigente la recomendación de intentar una aproximación laparoscópica antes de decidir convertir, donde se cuente con la experiencia y el equipo necesarios ${ }^{15,16}$. Se debe recordar que la conversión a cirugía abierta no es en ningún momento una complicación, sino un recurso válido que tiene el cirujano para llevar a cabo la cirugía de forma segura.

El otro punto para tener en cuenta es definir el tipo de colecistectomía subtotal que se realizará. Se han descrito múltiples técnicas para llevar a cabo dicho procedimiento; es importante tener en cuenta la perfusión de la pared vesicular y la claridad con que se vea el orificio cístico, en el momento de tomar la decisión de intentar alguna de las opciones. Nuestra técnica siempre fue de tipo reconstructiva, y cumple con los principios de dejar un muñón vesicular corto, extraer 
la totalidad de los cálculos y procurar la menor tasa de complicaciones posibles realizando una disección meticulosa. En ningún caso realizamos una técnica fenestrada.

Múltiples estudios han tratado de determinar los beneficios de una técnica sobre la otra; las diferencias encontradas al compararlas son una tendencia a mayor filtración e infección del sitio operatorio con la fenestrada, y una mayor frecuencia de eventos biliares y por ende cirugías adicionales con la reconstructiva, sin embargo, no han sido diferencias estadísticamente significativas ${ }^{9,10,13,16,17}$. El seguimiento más largo encontrado en la literatura fue de 6 años, sin diferencias significativas en la incidencia de estenosis, reintervenciones adicionales ni efectos negativos en la calidad de vida ${ }^{13,17}$.

En nuestra serie de casos, las características demográficas no varían significativamente con respecto a los demás pacientes con enfermedad biliar (principalmente mujeres, entre los 40 y 60 años). Ha sido motivo de investigación el momento óptimo para realizar una colecistectomía en el contexto urgente y algunos estudios han mostrado cómo la disección se facilita y la recuperación es más acelerada cuando se opera en la fase aguda al compararla con la subaguda (tiempo de síntomas mayor de 3 días). Es importante tener en cuenta que cuando los pacientes se presentaron a nuestro servicio de urgencias ya tenían en promedio 6,9 días de síntomas y esto se podría comportar como un factor preoperatorio para sospechar de antemano una colecistectomía difícil.

En nuestro grupo de pacientes tuvimos dos complicaciones importantes que ameritaron algún tipo de intervención para su manejo. Consideramos que estos resultados están dentro de lo esperado y que presentan un reto en su tratamiento ${ }^{\mathrm{I}, \mathrm{r}}$. Siempre que se considere realizar una colecistectomía subtotal, se está tratando de evitar una lesión de vía biliar al no tener claridad en las estructuras anatómicas. Aunque no se pueden sacar conclusiones con nuestros datos, presentamos una evolución y seguimiento de los pacientes en los cuales no se documentaron este tipo de lesiones, que generalmente causan gran morbilidad.
No a todos los pacientes en nuestro estudio se les dejó drenaje subhepático y en las historias no está claro el motivo. En cuanto a este tema, no hay un consenso al respecto, y en la literatura revisada se aprecia como su uso se basa más en la decisión del cirujano que en evidencia clínica que lo apoye. La técnica fenestrada, sería una indicación más fuerte hacia dejar el muñón del cístico ligado y el lecho quirúrgico drenado, pero como se mencionó, fue una técnica no utilizada en estos pacientes. Los autores tienen el concepto de que no es necesario dejar un dren en todos los casos, pero hacen énfasis en que pacientes con gangrena vesicular, donde hay un mayor riesgo de extensión de esta y de fístula postoperatoria, está indicado su uso ${ }^{13}$.

La estancia hospitalaria estuvo dentro de lo esperado al comparar los datos con otros reportes ${ }^{18-20}$. Nuestros pacientes en general fueron intervenidos en los primeros tres días posteriores al ingreso. Recomendamos realizar una cirugía temprana, evitando la progresión de la inflamación y de la infección, que dificultan técnicamente la disección. Respecto a la estancia postoperatoria, pese a estar dentro de lo descrito en la literatura, consideramos se pueden lograr tiempos menores, con altas hospitalarias más tempranas, apoyadas por sistemas de atención en casa que permitan completar el tratamiento antibiótico instaurado y vigilar el drenaje en los casos que se haya utilizado.

Nuestra tasa de conversión a cirugía abierta fue baja, similar a la reportada en otras series. Consideramos que esto no es una complicación de la cirugía, sino una decisión oportuna y válida al momento de enfrentar un paciente con inflamación severa que no permita una adecuada aproximación laparoscópica ${ }^{20}$. La decisión de convertir a cirugía abierta no tuvo que ver con el hecho de realizar una colecistectomía subtotal, sino que fue un hecho previo a tomar esta decisión.

La colecistectomía subtotal laparoscópica continúa siendo un procedimiento alternativo en pacientes con colecistectomía difícil, con una seguridad mayor y una tasa de complicación más baja. Como limitante del estudio, en el segui- 
miento a los pacientes operados, que fue limitado a la consulta ambulatoria postoperatoria y la revisión de la historia clínica de los pacientes para definir si hubo reingresos o reintervenciones, no se pueden definir con certeza las complicaciones a largo plazo. La principal desventaja de la técnica reconstructiva es la posibilidad de presentar cálculos en el remanente, nuevos o retenidos, que causen síntomas y requieran una nueva cirugía. La presencia de fístula biliar en el contexto agudo es variable y más frecuente en la técnica fenestrada; esta puede manejarse, bien sea de manera expectante esperando su cierre espontáneo, o de forma endoscópica mediante la CPRE.

Basado en lo anterior, es importante conocer las diferentes opciones existentes, así como sus ventajas y desventajas, lo que puede ayudar a reducir el porcentaje de complicación de esta cirugía, que alcanza el $40 \%{ }^{21}$. Los casos que presentaron fuga biliar fueron manejados de forma exitosa mediante endoscopia o cirugía mínimamente invasiva, sin complicaciones adicionales. Esto se traduce en un II, $7 \%$ de reintervenciones, cifra elevada pero que consideramos está dentro de lo esperado en casos difíciles como los que se describen.

\section{Conclusión}

La colecistectomía difícil continúa siendo una de las indicaciones más frecuentes para conversión a cirugía abierta. En la serie de casos presentada, mostramos nuestros resultados con la colecistectomía subtotal. A pesar de que su indicación no es rutinaria, el cirujano deberá tener conocimiento de las diferentes técnicas quirúrgicas para poder implementarlas cuando lo considere necesario. Queda el interés, partiendo de estudios descriptivos y retrospectivos como este, para realizar a futuro estudios con mayor número de pacientes y un seguimiento más largo, que permitan sacar conclusiones con mejor nivel de evidencia.

Agradecimientos: Por la asesoría epidemiológica a Martha Ofelia Correa, médica especialista en Cirugía Vascular y magister en Epidemiología Clínica.

\section{Cumplimiento de normas éticas}

Consentimiento informado: Este estudio es una revisión de historias clínicas retrospectiva, y como tal, no hay necesidad de un consentimiento informado. El Comité de Ética Institucional aprobó el diseño y la metodología del estudio.

Conflicto de interés. No hay conflictos de intereses declarados.

Financiación. Autofinanciación.

\section{Referencias}

I. Elwood DR. Cholecystitis. Surg Clin North Am. 2008;88:I24I-52, viii.

https://doi.org/IO.IoI6/j.suc.2008.07.008

2. Gaitán JA, Martínez VM. Enfermedad litiásica biliar, experiencia en una clínica de cuarto nivel, 2005-20II. Rev Colomb Cir. 2017;29:188-96.

3. Shaffer EA. Gallstone disease: Epidemiology of gallbladder stone disease. Best Pract Res Clin Gastroenterol. 2006;20:98I-96. https://doi.org/IO.IOI6/j.bpg.2006.05.004

4. Stinton LM, Myers RP, Shaffer EA. Epidemiology of Gallstones. Gastroenterol Clin North Am. 2010;39:157-69. https://doi.org/IO.IOI6/j.gtc.20I0.02.003

5. Coccolini F, Catena F, Pisano M, Gheza F, Fagiuoli S, Di Saverio S. Open versus laparoscopic cholecystectomy in acute cholecystitis. Systematic review and meta-analysis Int J Surg. 2015;18:196-204. https://doi. org/IO.IoI6/j.ijsu.2015.04.083

6. Gourgiotis S, Dimopoulos N, Germanos S, Vougas V, Alfaras P, Hadjiyannakis E. Laparoscopic cholecystectomy: a safe approach for management of acute cholecystitis. JSLS. 2007;II:219-24

7. Henneman D, da Costa DW, Vrouenraets BC, van Wagensveld BA, Lagarde SM. Laparoscopic partial cholecystectomy for the difficult gallbladder: a systematic review. Surg Endosc. 20I3;27:35I-8. https://doi. org/IO.IOO7/s00464-0I2-2458-2

8. Strasberg SM, Brunt LM. Rationale and use of the critical view of safety in laparoscopic cholecystectomy. J Am Coll Surg. 20I0;2II:I32-8. https://doi.org/IO.IOI6/j. jamcollsurg.2010.02.053

9. Strasberg SM, Pucci MJ, Brunt LM, Deziel DJ. Subtotal cholecystectomy-"fenestrating" vs «reconstituting» subtypes and the prevention of bile duct injury: Definition of the optimal procedure in difficult operative conditions. J Am Coll Surg. 2016;222:89-96. https://doi. org/IO.IOI6/j.jamcollsurg.2015.09.019

Io. Elshaer M, Gravante G, Thomas K, Sorge R, Al-Hamali S, Ebdewi H. Subtotal cholecystectomy for «difficult 
gallbladders»: systematic review and meta-analysis. JAMA Surg. 20I5;150:159-68. https://doi.org/IO.IOoI/jamasurg.20I4.I219

II. Álvarez LF, Rivera D, Esmeral ME, García MC, Toro DF, Rojas OL. Colecistectomía laparoscópica difícil, estrategias de manejo. Rev Colomb Cir. 2013;28:186-95.

12. Abdelrahim WE, Elsiddig KE, Wahab AA, Saad M, Saeed H, Khalil E. Subtotal laparoscopic cholecystectomy influences the rate of conversion in patients with difficult laparoscopic cholecystectomy: Case series. Ann Med Surg. 20I7;19:I9-22. https://doi.org/IO.IOI6/j. amsu.2017.04.018

I3. van Dijk AH, Donkervoort SC, Lameris W, de Vries E, Eijsbouts QAJ, Vrouenraets BC, et al. Short- and longterm outcomes after a reconstituting and fenestrating subtotal cholecystectomy. J Am Coll Surg. 20I7;225:37I-9. https://doi.org/IO.IOI6/j.jamcollsurg.20I7.05.016

I4. Ji W, Li LT, Li JS. Role of laparoscopic subtotal cholecystectomy in the treatment of complicated cholecystitis. Hepatobiliary Pancreat Dis Int. 2006;5:584-9.

15. Kim Y, Wima K, Jung AD, Martin GE, Dhar VK, Shah SA. Laparoscopic subtotal cholecystectomy compared to total cholecystectomy: a matched national analysis. J Surg Res. 2017;218:316-2I. https://doi.org/IO.IOI6/j. jss.2017.06.047

I6. Kirkwood R, Damon L, Wang J, Hong E, Kirkwood K. Gangrenous cholecystitis: innovative laparoscopic techniques to facilitate subtotal fenestrating cholecystec- tomy when a critical view of safety cannot be achieved. Surg Endosc. 20I7;3I:5258-66. https://doi.org/IO.IOO7/ s00464-017-5599-5

I7. Soleimani M, Mehrabi A, Mood ZA, Fonouni H, Kashfi A, Büchler MW, et al. Partial cholecystectomy as a safe and viable option in the emergency treatment of complex acute cholecystitis: A case series and review of the literature. Am Surg. 2007;73:498-507. https://doi. org/I0.II77/000313480707300516

I8. Supit C, Supit T, Mazni Y, Basir I. The outcome of laparoscopic subtotal cholecystectomy in difficult cases - A case series. Int J Surg Case Rep. 20I7;4I:3II-4. https://doi. org/Io.IoI6/j.ijscr.20I7.IO.054

19. Jara G, Rosciano J, Barrios W, Vegas L, Rodríguez O, Sánchez R, et al. Colecistectomía laparoscópica subtotal como alternativa quirúrgica segura en casos complejos. Cirugía Española. 2017;95:465-70. https://doi. org/IO.IOI6/j.ciresp.2017.07.013

20. Ashfaq A, Ahmadieh K, Shah AA, Chapital AB, Harold KL, Johnson DJ. The difficult gall bladder: Outcomes following laparoscopic cholecystectomy and the need for open conversion. Am J Surg. 2016;212:I26I-4. https://doi.org/Io.IoI6/j.amjsurg.20I6.09.024

2I. Lidsky ME, Speicher PJ, Ezekian B, Holt EW, Nussbaum DP, Castleberry AW, et al. Subtotal cholecystectomy for the hostile gallbladder: failure to control the cystic duct results in significant morbidity. HPB. 20I7;19:547-56. https://doi.org/IO.IOI6/j.hpb.20I7.02.44I 